he genetic method is by its nature analytic, so it is most effectively employed in partnership with the complementary disciplines of systems biology and physiology, which can reconstruct the normal functioning of parts encoded in the genome. Experts in the latter field are often available in the form of clinical researchers specializing in a particular organ system. Incorporating new genetic knowledge is then a matter of putting the newly identified pieces of the human body into their places, one organ system at a time. Indeed, this is the process we hope to stimulate with our conference on nephrogenetics that takes place early this month in Danvers, Massachusetts, organized in collaboration with the International Society for Nephrology (http://www.associationhq.com/ISN/forefronts/).

The meeting is built around the remarkable body of insights into renal development and function that we have learned from researchers studying mendelian disorders of the human kidney. Many such studies have been published in this journal, and some examples of recent papers are freely available at http://www. connotea.org/user/Dicentrarchus0labrax/tag/nephrology. This knowledge is augmented by genetic studies from a panoply of model organisms. By bringing together the genetic evidence that dissects both the development and adult physiological function of this one organ system, we hope to create for meeting participants a unified view of the kidney at the level of organism, organ and organelle (see Nat. Genet. 38, 131; 2006). If we are successful in synthesizing a new integrated view of kidney development and function based on the roles of single genes, the next challenge will be to assemble a comprehensive overview of the contributions of genetic variants to common and complex renal diseases. Of necessity, this will entail consideration of related physiological systems in which the kidney has a major role (as in hypertension, diabetes and cardiovascular disease).

At the same time that our meeting looks inward to the operation of renal systems, the major renal societies are looking outward with a publicity campaign called World Kidney Day. Their message is that kidney disease is common (affecting about $10 \%$ of the population worldwide) and that we already know enough to save many lives for not much money using existing diagnostics and treatments. Furthermore, implementation of these strategies will have worldwide benefits, and it might even be more cost effective in less well-resourced countries (see Nat. Genet. 37, 927; 2005).

Why is it so hard to implement existing medical knowledge? Are many people unaware of what kidneys do and what kidneys can suffer from? For one thing, end-stage renal disease is relatively uncommon, affecting about 1 of 500 people in countries that can offer therapy for failing kidneys. Second, being diseases with gradual onset, hypertension and chronic kidney disease are most dramatically recognized by their final consequences, when they result in cardiovascular emergencies. So, they may not be widely perceived as 'real' diseases by the general public.

Genetics is a great tool for new discoveries and a splendid way to bring attention to the function of organelles, organs and organisms. Sometimes, as on March $8^{\text {th }}$, World Kidney Day, it would be good to take a minute to check that what we have learned as geneticists is getting through to the people who trust researchers to deliver better healthcare. 


\section{ERRATUM: Nephrogenetics on World Kidney Day}

Nat. Genet. 39, 277 (2007); published online 28 February; corrected after print 2 March 2007

In the version of this article initially published, a reference citation was incorrect: Nat. Genet. 38, 131 (2006) should have been cited instead of Nat. Genet. 39, 133 (2007). The error has been corrected in the HTML and PDF versions of the article. 